\title{
Structure and dynamics of the interface between a binary hard-sphere crystal of NaCl type and its coexisting binary fluid
}

\author{
Rachel Sibug-Aga and Brian B. Laird* \\ Department of Chemistry, University of Kansas, Lawrence, Kansas 66045
}

(Received 28 May 2002; published 15 October 2002)

\begin{abstract}
Molecular-dynamics simulations are performed to study the [100] and [111] orientations of the crystal-melt interface between an ordered two-component hard sphere with an $\mathrm{NaCl}$ structure and its coexisting binary hard-sphere fluid. The diameter ratio of the two types of hard spheres making up the mixture is $\alpha=0.414$. This work complements our earlier interface simulations [J. Chem. Phys. 116, 3410 (2002)] for the same diameter ratio at lower pressures where the smaller component is immiscible in the solid and the fluid mixture coexists with a pure fcc crystal of large particles. Density profiles and diffusion coefficient profiles are presented for the $A B$ interfacial system. We find that for this system, the transition from crystallike to fluidlike behavior of both the density and diffusion constant profiles occurs over a narrower region than that seen in our previous studies of the fcc/binary fluid system. But similar to what was found in the fcc/binary fluid interface the transition region for the large particle diffusion constant is shifted about $1.0 \sigma_{A}$ toward the fluid phase relative to that for the small particles.
\end{abstract}

DOI: 10.1103/PhysRevB.66.144106

PACS number(s): 68.08.De

\section{INTRODUCTION}

The kinetics of crystal growth and nucleation from the melt is highly dependent upon the structure, dynamics and thermodynamics of the crystal-melt interface. ${ }^{1}$ Given the difficulties in obtaining unambiguous information from experiments, most of what is currently known about the microscopic phenomenology of such interfaces is obtained via computer simulation. ${ }^{2}$ Although most simulation studies have focused on single component systems, ${ }^{3-10}$ there has been recent interest in multicomponent interfaces. ${ }^{11-14}$ All of these studies have involved crystal phases that are either disordered or pure face-centered cubic (fcc) lattices. In this work we present results for the structure and dynamics of the interface between an ordered two-component hard-sphere crystal with a sodium chloride $(\mathrm{NaCl})$ structure and a binary hard-sphere fluid. Such a system can be viewed either as a prototype to understand the interface between intermetallic compounds and their coexisting fluid phases or as a model two-component colloidal dispersion.

The hard-sphere interaction was chosen for this study since it is an important reference model for the study of simple liquids ${ }^{15}$ and liquid mixtures. ${ }^{16}$ This is especially true with regard to phenomena associated with the freezing transition. For example, it has been recently shown that the interfacial free energy of close-packed metals can be described with quantitative accuracy using a hard-sphere model. ${ }^{17}$ In addition, recent phase boundary calculations have shown that binary hard spheres form a wide range of crystal structures depending on the ratio $\alpha=\sigma_{B} / \sigma_{A}$ of the small sphere (type $B$ ) diameter $\sigma_{B}$ to that of the larger spheres (labeled $A$ ) $\sigma_{A}$. A substitutionally disordered fcc crystal is the stable phase for $1.0>\alpha>0.85$ (Ref. 18) while for $\alpha<0.85$, only ordered crystal structures are seen to be stable, including $A B, A B_{2}$, and $A B_{13}$ structures. ${ }^{19-23}$ A detailed study of the disordered fcc crystal/melt interface for $\alpha=0.9$ has been recently reported. ${ }^{12}$
In this work, we examine two-component hard-sphere mixtures with a considerably larger size asymmetry of $\alpha$ $=0.414$. This size ratio is significant in the theory of binary alloys in that it is the largest asymmetry in which the small spheres can be accommodated in the interstitials of a densest close-packed crystal of larger spheres. The phase diagram for this value of $\alpha$ has been determined as a function of pressure and mole fraction using $\mathrm{MC}$ and $\mathrm{MD}$ simulations by Trizac and co-workers ${ }^{19}$ and is reproduced in Fig. 1. At low pressures, the binary fluid coexists with a pure fcc crystal of large spheres, whereas at higher pressures (above $50 k T / \sigma_{A}^{3}$ ) the coexisting solid phase is an ordered 1:1 crystal of the sodium

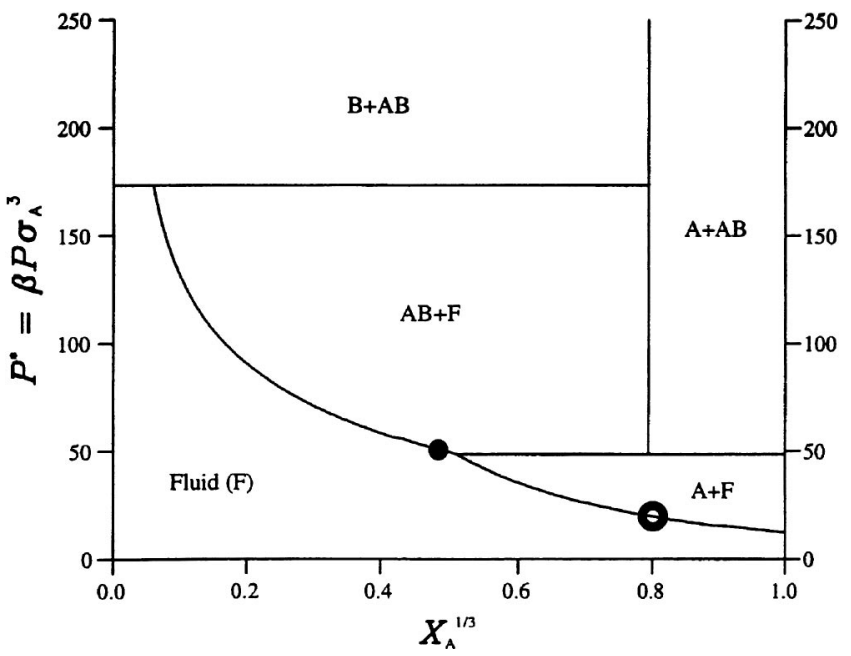

FIG. 1. Pressure-concentration phase diagram of the binary hard-sphere system with $\alpha=0.414$. [Reprinted from Ref. 19 by permission of the publisher, Taylor and Francis, Ltd. (www.tandf.co.uk/journals)]. Note that to make the phase coexistence lines easier to distinguish, the pressure is plotted against the cube root of the large sphere mole fraction. The phase point of the present study is shown as a filled circle. The open circle shows the conditions of our previous study (Ref. 13). 
chloride $(\mathrm{NaCl})$ type. Earlier cell theory calculations also predicted the stability of the $\mathrm{NaCl}$ at this diameter ratio. ${ }^{20}$ Other $A B$ structures such as the $\mathrm{CsCl}$ and the zinc blende have been shown to be unstable at this diameter ratios. ${ }^{20,23}$ Throughout the text we will be using $A B$ to also refer to the $\mathrm{NaCl}$ structure.

A detailed study of the low pressure pure fcc/binary fluid system has been recently reported. ${ }^{13}$ In that work, the coexistence between the crystal and an approximately 1:1 binary mixture was examined, corresponding to a pressure of $20.1 k T / \sigma_{A}^{3}$, which is approximately twice the coexistence pressure $(11.55 \mathrm{kT} / \sigma)$ of the pure single component system. As the pressure is increased the mole fraction of large spheres in the fluid phase $X_{A}^{f}$ decreases, and at a pressure of about $50 \mathrm{kT} / \sigma_{A}^{3}$ the fluid coexists with an $A B$ crystal of $\mathrm{NaCl}$ type (see Fig. 1). In this work, we examine in detail the microscopic structure and dynamics of the interface between the high pressure $A B$ crystal and its melt. To do this, we have chosen a point in the phase diagram with $X_{A}^{f}=0.097$. At this mole fraction, the fluid coexists with the $\mathrm{NaCl}$ crystal at a pressure of $53 k T / \sigma_{A}^{3}$. Details of the simulation methodology and interface equilibration procedure are outlined in the next section followed by presentation of the results of the study in Sec. III and concluding remarks in Sec. IV.

\section{SIMULATION DETAILS}

Molecular-dynamics simulations are performed on a twocomponent system of hard spheres of differing diameters $\sigma_{A}$ and $\sigma_{B}$, with $\sigma_{A}>\sigma_{B}$ (type $A$ particles are assumed to be the larger spheres). The interaction potential between two spheres is defined by the following pairwise interaction potential:

$$
\phi_{i j}(r)= \begin{cases}\infty, & r \leqslant \sigma_{i j}, \\ 0, & r>\sigma_{i j},\end{cases}
$$

where $i, j \in\{A, B\}, r$ is the distance between the centers of the two interacting spheres, and $\sigma_{i j}=\left(\sigma_{i}+\sigma_{j}\right) / 2$ is the distance of closest possible approach between two spheres with diameter $\sigma_{i}$ and $\sigma_{j}$, respectively. The system is completely defined by specifying the diameter ratio $\alpha \equiv \sigma_{B} / \sigma_{A}$, the mole fraction of large particles, $x_{A}$ and the total number density $\rho$. The total volume occupied by the hard spheres relative to the volume available to the system is given by the packing fraction

$$
\eta=\frac{\pi \sigma_{A}^{3}}{6} \rho\left[x_{A}+\left(1-x_{A}\right) \alpha^{3}\right],
$$

where $\rho=\rho_{A}+\rho_{B}=N / V$ is the total density, $x_{A}$ is the mole fraction of the larger species, and $\alpha=\sigma_{B} / \sigma_{A}$ is the diameter ratio.

The procedures for interface construction and equilibration of binary interfaces employed in this study are similar to those used in our earlier work ${ }^{13}$ on the low-pressure coexistence in this system [single component fcc $\left(x_{A}=1\right) /$ binary fluid mixture]. Since the general interface preparation process is described at length in that work, only those details specific to the current system are described here. The interested reader is encouraged to consult Ref. 13 for a more complete description.

To construct an interface, fluid and crystal blocks are prepared separately using the calculated coexistence conditions. At a pressure of $53 k T / \sigma_{A}^{3}$, we independently determined the packing fractions of the coexisting crystal and fluid to be $\eta^{c}=0.683$ and $\eta^{f}=0.490$, respectively. In the preparation of a fluid block, it is a usual practice to initially position particles in a lattice at a density lower than coexistence. As the system is allowed to equilibrate the lattice melts, giving a fluid configuration, which is then compressed to the coexistence density. For the system under study here this procedure is not feasible since the size asymmetry $(\alpha=0.414)$ and the mole fraction of small particles ( $\left.X_{B}^{f}=0.903\right)$ in the fluid system are both large. (It should be noted that for this value of $\alpha$ the large sphere volume is over 14 times that of the small particle.) To construct the fluid phase we began with an fcc lattice of small particles at a number density equal to the desired total number density of the fluid mixture. A number of particles, corresponding to the target mole fraction of large spheres, are then chosen at random from this lattice. A molecular dynamics run is started from this initial configuration and the diameter of randomly chosen particles is periodically increased until the correct large sphere diameter is attained. The amount of increase in the diameter at each stage depends on the maximum increase that is possible without creating particle overlap. The preparation of the crystal is straightforward as the small particles easily inserted into the interstitial sites of the large sphere fcc lattice.

After equilibration of separate crystal and fluid systems, they are placed in contact within the simulation box. Due to the periodic boundary conditions, two interfaces are formed. Note that packing fraction used in the preparation of the initial fluid block is slightly different from the predicted $\eta^{f}$ because a gap of $1 \sigma_{A}$ is placed between the crystal and fluid blocks to avoid any initial overlap that may occur when the two blocks are combined. The fluid particles are then allowed to move while the large particles are fixed so the initial gaps are then filled with the fluid particles. The initial fluid packing fraction is adjusted until an unstressed bulk crystal is obtained when the two blocks are combined and equilibrated

In this study, we examine both the [100] and [111] orientations of this $\mathrm{NaCl}$ crystal/binary fluid interface. For reference, we define the $z$ axis to be perpendicular to the interfacial plane. Periodic boundary conditions are applied in $x, y$, and $z$ directions. The length in the $z$ direction $L_{z}$ is a sum of lengths of the two separate blocks in the $z$ direction and the $2 \sigma_{A}$ gap initially left between the two blocks. The total number of particles used are 22032 and 22752 for the [100] and [111] orientations, respectively. The details of the system sizes used are summarized in Table I. As the systems studied are large, we have implemented the cell method technique ${ }^{24}$ to efficiently carry out the molecular-dynamics simulations. Equilibration was done for about $4 \times 10^{5}$ collisions per particle (cpp). During sampling, the run was divided into blocks of $2600 \mathrm{cpp}$ each. The sampling run was over a length of 30 blocks, but since each block gives two independent measures 
TABLE I. Number of particles and dimensions of the simulation box.

\begin{tabular}{lccccc}
\hline \hline & $N^{c}$ & $N^{f}$ & $L_{x} / \sigma_{A}$ & $L_{y} / \sigma_{A}$ & $L_{z} / \sigma_{A}$ \\
\hline$[100]$ & 7056 & 14976 & 10.41 & 10.41 & 53.02 \\
{$[111]$} & 7200 & 15552 & 10.51 & 10.92 & 51.44 \\
\hline \hline
\end{tabular}

of interfacial properties when the system is properly folded at the center of the crystal, the results reported are averages taken over 60 samples.

In the course of such simulations the position of the interfacial plane can shift due to Brownian motion or due to melting or freezing of the crystal. To prevent such motion from artificially broadening the interfacial profiles it is necessary to monitor the position of the interfacial plane during the sampling runs. The standard measure of interfacial position for a planar interface is the Gibbs dividing surface ${ }^{1}$ defined for a multicomponent system as the value of $z$ where the surfaces excess number of "solvent" particles is zero. However, accurate calculation of the Gibbs dividing surface requires relatively long simulation runs and is then unsuitable for a method of monitoring the time dependence of interfacial position. In our previous studies, ${ }^{7,13}$ we find that a more suitable measure can be obtained from measurement of orientational order parameter profile as a function of $z$. The orientational order parameter is defined as follows:

$$
q_{n}(z)=\left\langle\frac{1}{N_{z}} \sum_{i, j, k} \cos \left[n \theta_{x y}(i, j, k)\right]\right\rangle,
$$

where $n=4$ for the [100] orientation and $n=6$ for the [111], $i, j$, and $k$ are nearest neighbor large particles, $\theta_{x y}(i, j, k)$ is the bond angle formed by $i, j$, and $k$ projected on the $x, y$ plane, and $N_{z}$ is the total number of particles that form bond angles. The average is taken over the number of angles found between $z-\Delta z / 2$ and $z+\Delta z / 2$, where $\Delta z$ is equal to the layer spacing of the bulk crystal.

We show in Fig. 2 the order parameters $q_{4}$ and $q_{6}$ of the two particle types for the [100] (upper panel) and [111] (lower panel) orientations. As expected $q_{4}\left(q_{6}\right)$ is small in the [111] ([100]) interface where sixfold (fourfold) symmetry dominates. We define the interfacial position relative to the midpoint of the orientational order profile for the large particles. That is, $z=0$ in all of the $z$-dependent profiles presented in this study is defined as the point at which the orientational order parameter has decayed halfway from its crystal to fluid value. This order parameter is suitable as a measure of interfacial position since it is smoothly monotonically decreasing and can be calculated accurately for very short runs. The parameter profiles of the small particles are not smoothly varying because at the interfacial region, some number of small particles cluster together to occupy large particle vacancies at the interfacial region (as will be seen in the density plots presented in the next section) disrupting the smooth transition from crystal-like to fluid-like value of the orientational order parameter.

Analysis of the interfacial position as a function of time shows that during the equilibration run the crystal exhibits

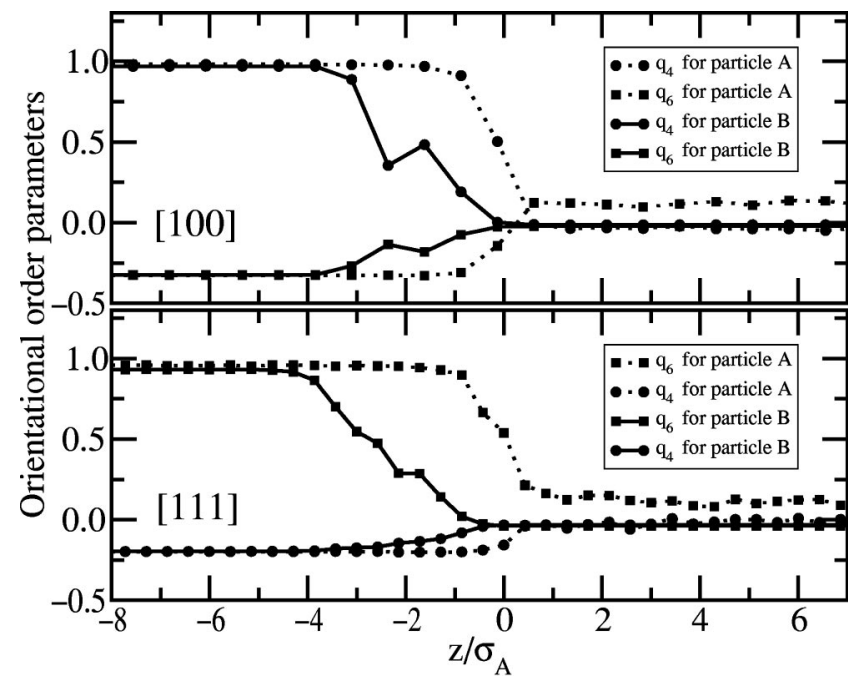

FIG. 2. Large (dotted) and small (solid) particle orientational order parameter profiles, $q_{4}$ (circle) and $q_{6}$ (square) for the [100] and [111] interfacial orientations. The point $z=0$ is the location of the interfacial plane as calculated from the large particle order parameter profile.

some initial growth, but quickly stabilizes before the averaging runs are begun. Brownian motion of the solid phase, as monitored by motion of the inner layers of the crystal, was found to be negligible due to the large system size and no correction was necessary.

\section{SIMULATION RESULTS FOR THE [100] AND [111] INTERFACES}

A. Structure: Density profiles and contour plots

The structural variation of the system across the interface is determined by calculating the density profile for each particle type:

$$
\rho_{i}(z)=\frac{\left\langle N_{i}(z)\right\rangle}{L_{x} L_{y} \Delta z}
$$

where $i$ denotes a particle type, $\Delta z$ is $1 / 25$ of the crystal layer spacing, $\left\langle N_{i}(z)\right\rangle$ is the average number of particles of type $i$ in the region between $z-\Delta z / 2$ and $z+\Delta z / 2$.

The density profiles of the two particle types are shown in Figs. 3(a) and 3(b) for the [100] and [111] orientations, respectively. As expected for an $\mathrm{NaCl}$-type lattice, the small and large particle peaks are in phase in [100] and exactly out of phase in [111]. This is similar to the registry of particle density peaks found at the interfacial region of the lower pressure pure fcc/binary fluid system. ${ }^{13}$ Due to the higher pressures in this study, the crystal peaks are much sharper than those seen in the lower pressure binary system ${ }^{13}$ or in the single component interface. ${ }^{7}$ The small side peaks in the $\mathrm{NaCl}$ density are due to the filling of large particle vacancies in the lattice structure with several smaller particles-as discussed below.

In order to reveal any change in the lattice spacing through the interface and to index the interfacial planes for later use, vertical dotted lines separated by the bulk crystal 


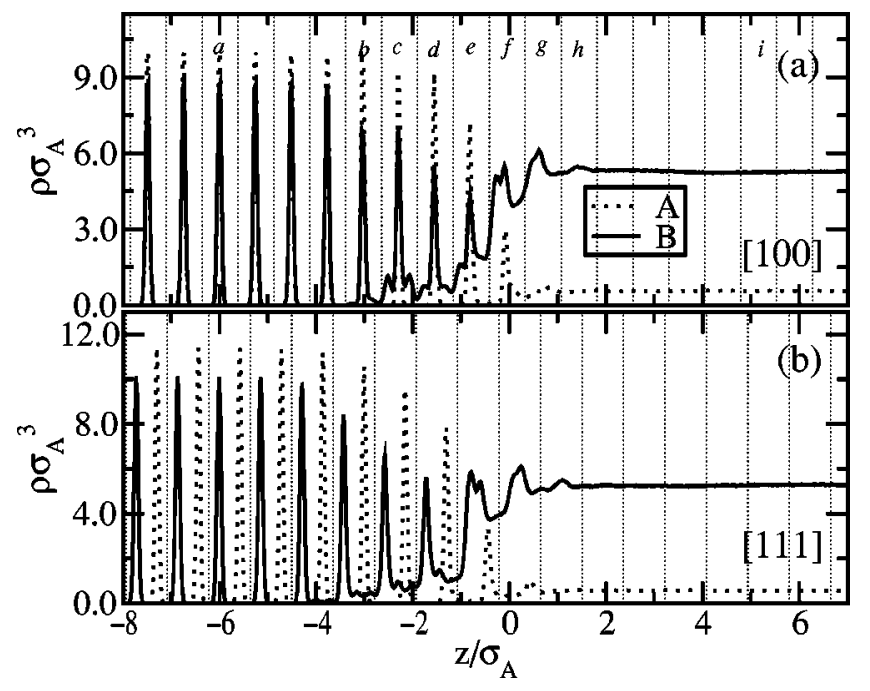

FIG. 3. Large (dotted) and small (solid) particle density profiles of the $\mathrm{NaCl} /$ binary fluid interface for the [100] and [111] orientations. The distance between vertical dotted lines is equal to the crystal layer spacing in [100] and twice the spacing in [111].

lattice spacing were added to Fig. 3. Labels $a$ to $i$ in the [100] profile mark specific layers for which cross-sectional density distributions have been determined, as discussed later. For both orientations, there is no discernible variation in the lattice spacing as the interface is traversed from crystal to fluid, in contrast to what was seen in our previous interface studies with either pure or disordered fcc crystals ${ }^{7,12,13}$ which exhibit a significant increase in the density peak spacing for the [100] orientation as the fluid side of the interface is approached. In those systems the presence of the interface induces significant structure in the fluid, but as one moves further away from the interface into the fluid the influence of the specific crystal orientation diminishes and the peak spacing in the fluid reverts to the spacing that would be formed at a structureless wall. Since this natural spacing is nearly identical to the [111] crystal layer spacing, there is minimal change in the peak spacing for that orientation. However, the layer spacing in a [100] orientation is about $15 \%$ smaller than the "natural" value and a gradual increase is observed.

To better understand the lack of variablity of the lattice spacing for the $\mathrm{NaCl} /$ binary fluid interface under current study, we have performed separate Monte Carlo (MC) simulations for binary hard-sphere fluids at a structureless hard wall, using densities and mole fractions corresponding to both the coexisiting fluid phase of the current study and that of our previous examination of the lower pressure pure fcc/ binary fluid interface. The MC simulations are performed on 648 particles, equilibrated for $1 \times 10^{6}$ cycles with density profiles averaged over $2 \times 10^{6}$ cycles. Figure 4(a) shows the result for the $1: 1$ binary fluid $(\eta=0.51)$ that coexists with the fcc crystal. The figure shows a peak spacing of $0.9 \sigma_{A}$, which is identical to the peak spacing that was observed in the single component system, ${ }^{7}$ indicating that the peak spacing in this system is determined by the large particle size. Thus, for an interface between such a fluid and a pure fcc crystal, the peak spacing on both sides of the interface is controlled by the large particle spacing and will smoothly
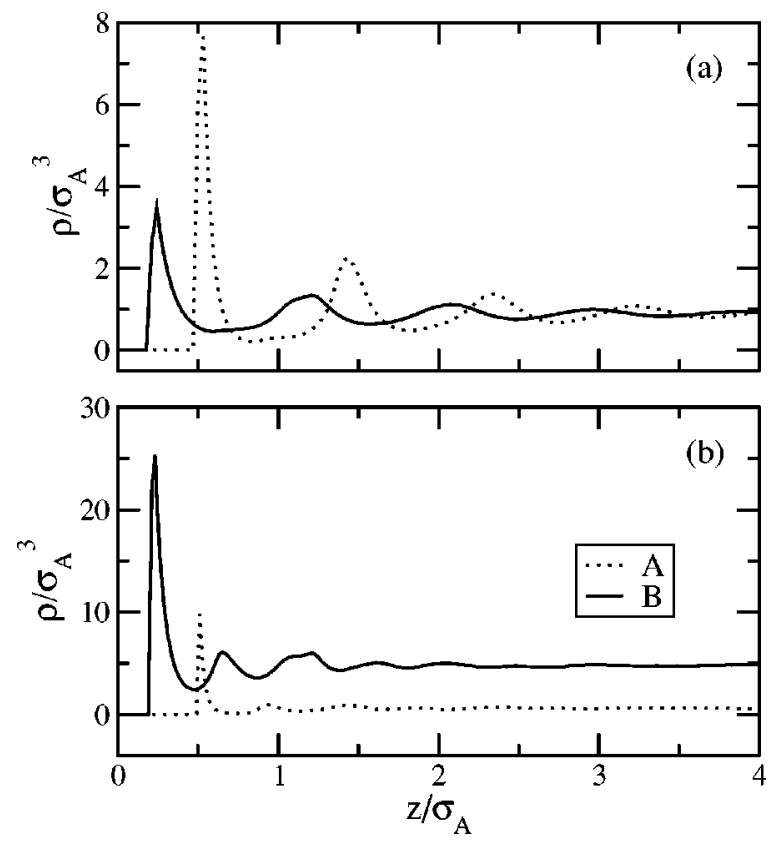

FIG. 4. Structure of binary fluids with a diameter ratio of $\alpha$ $=0.414$ against a structureless wall: (a) $x_{A}=0.5$ and $\eta=0.51$; (b) $x_{A}=0.1$ and $\eta=0.49$.

interpolate between the natural crystal and fluid values, as is observed in simulation. ${ }^{13}$ However, when the binary fluid coexisting with the NaCl-type crystal is placed next to a structureless hard wall, the density oscillations have much smaller density peak spacings that range from $0.42 \sigma_{A}$ to $0.48 \sigma_{A}$ for both particle types, as shown in Fig. 4(b). Since this is close to the diameter ratio of 0.414 , the peak spacing in this system is controlled by the small particle diameter. In addition, the range of influence of the wall is considerably shorter for the high pressure $\mathrm{NaCl}$ coexistence than that for the pure FCC system. In contrast, the layer spacing in the coexisting $\mathrm{NaCl}$ crystal $\left(0.74 \sigma_{A}\right.$ for [100] and $0.86 \sigma_{A}$ for [111]) is still determined by the large particle spacing. The large difference between the natural length scales in the crystal and fluid prevents a smooth transition between the two limits and only one length scale can be present in the interface. This dominant length scale must be that of the crystal, since the packing geometry prevents the crystal from adopting the smaller length scale of the fluid.

The oscillations in the fine scale density profiles shown in Fig. 3 make it difficult to see the overall trend in bulk density, so we have processed these profiles using a finite impulse response filter ${ }^{25,7}$ to reveal the nonoscillatory component of the density variation. The resulting filtered density profiles are shown in Fig. 5. The 10-90 width of these bulk density profiles provides a measure of the interfacial width. (The 10-90 width of a monotonically varying interfacial profile is the distance over which the profile changes from 10 to $90 \%$ of the higher of two coexisting bulk values, relative to the lower bulk value.) The 10-90 width derived from the large particle density profile of the [100] orientation [see Fig. $5(\mathrm{a})]$ is $2.6 \sigma_{A}$, corresponding to the region between $z$ $=-2.2 \sigma_{A}$ to $z=0.4 \sigma_{A}$. For the small particles the $10-90$ width is smaller at $2.3 \sigma_{A}$ and the 10-90 region ( $z$ 


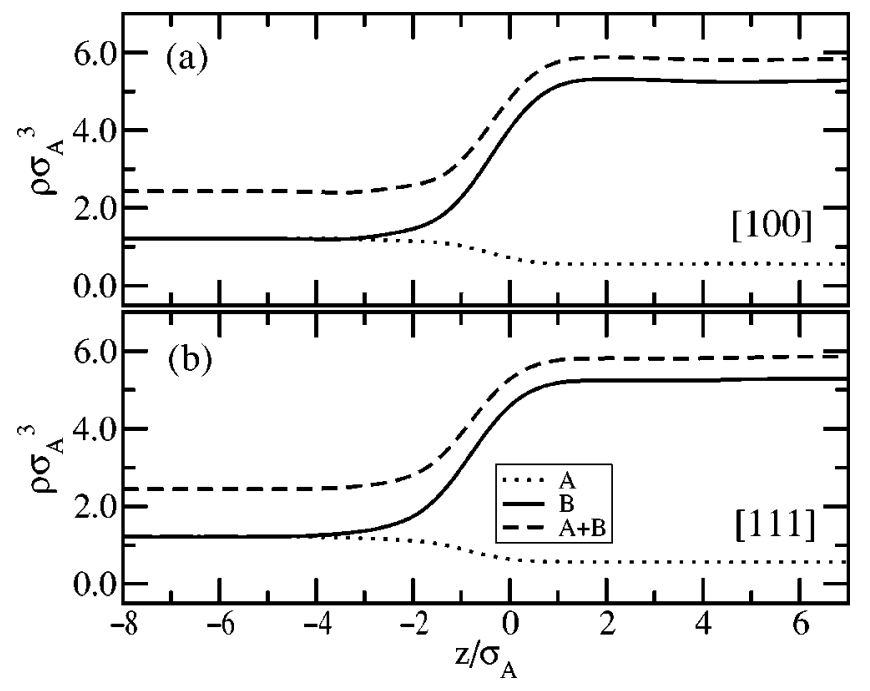

FIG. 5. Filtered density profiles for the large (dotted), small (solid), and total (dashed) densities in the (a) [100] and (b) [111] interfacial orientations.

$=-1.7 \sigma_{A}$ to $z=0.6 \sigma_{A}$ ) is shifted slightly toward the fluid, relative to the 10-90 region of the large spheres. Combining these two regions, the interfacial region of the [100] orientation defined by the densities has a width of $2.8 \sigma_{A}$. The total interfacial width defined for the densities for the [111] orientation $\left(2.9 \sigma_{A}\right)$ is not significantly different than that for [100]. The interfacial widths of the large particle density profiles are identical to those found in our earlier lower pressure fcc/binary fluid interface study ${ }^{13}$ for the same diameter ratio, but narrower than the $3.3 \sigma$ widths found in the single component system. ${ }^{7}$ In contrast, the small particle interfacial widths found here are much smaller than those found in the lower pressure study, where they were found to be about $3.3 \sigma_{A}$. As a consequence, the overall interface for the $\mathrm{NaCl} /$ fluid is slightly narrower than the lower pressure pure fcc/ fluid interface.

To get a detailed understanding of the structural transition across the interface between the two coexisting phases, we have determined the cross-sectional density distributions within layers parallel to the interfacial plane. For each layer, the $2 \mathrm{D}$ density distribution is defined as

$$
\rho_{i}^{z}(x, y)=\frac{\left\langle N_{i}^{z}(x, y)\right\rangle}{\Delta x \Delta y \Delta z},
$$

where $i$ denotes a particle type, $\Delta x=\Delta y=0.12 \sigma_{A}, \Delta z$ is the crystal layer spacing, which is $0.74 \sigma_{A}$ for [100]. The average number of particles of type $i$ in the volume given by $\Delta x \Delta y \Delta z$ is $\left\langle N_{i}^{z}(x, y)\right\rangle$. In Figs. 6 and 7 are density contour plots of the [100] interface for the large and small particles, respectively. Layers $a$ to $i$ are as labeled in Fig. 3(a), where $a$ is deep into the bulk crystal, $i$ is in the bulk fluid and $b$ to $h$ are interfacial regions. The decrease in density peak height of the large (type $A$ ) spheres in Fig. 3 starting at layer $b$ is initially due to the presence of lattice vacancies as shown in Fig. 6. Small particle vacancies also start to appear in layer $b$ of Fig. 7. We also find by comparing Figs. 3 and 6 for layers $c$ and $d$ that the side peaks appearing at these layers are due
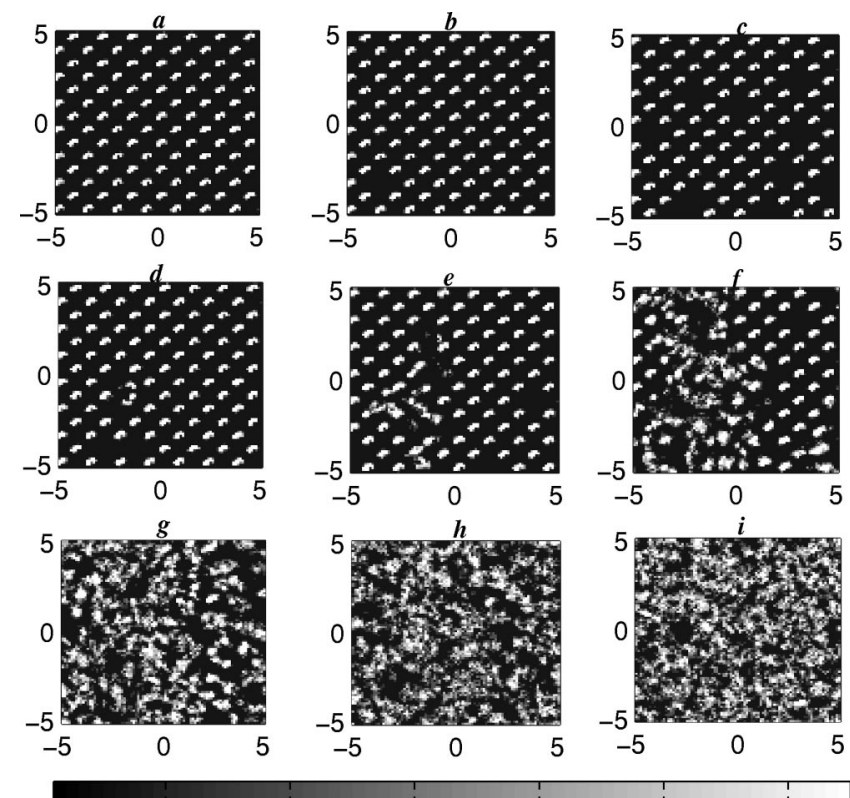

10

20

30

40

50

60

FIG. 6. Large particle density contour plots parallel to the interfacial plane for different layers of the [100] interface. The layers are as labeled in Fig. 3(a).

to the accumulation of small particles in the large sphere vacancies. The structure of this vacancy filling is interesting in that the large particle is typically replaced by 6 small particles (although a small number of vacancies filled with 5 or 7 small spheres do occur) with little disturbance to the surrounding lattice. This can be seen in Fig. 8 where a snapshot of one of these filled vacancies in layer $c$ is shown. A uniform $2 \mathrm{D}$ density distribution begins to develop in layer $g$
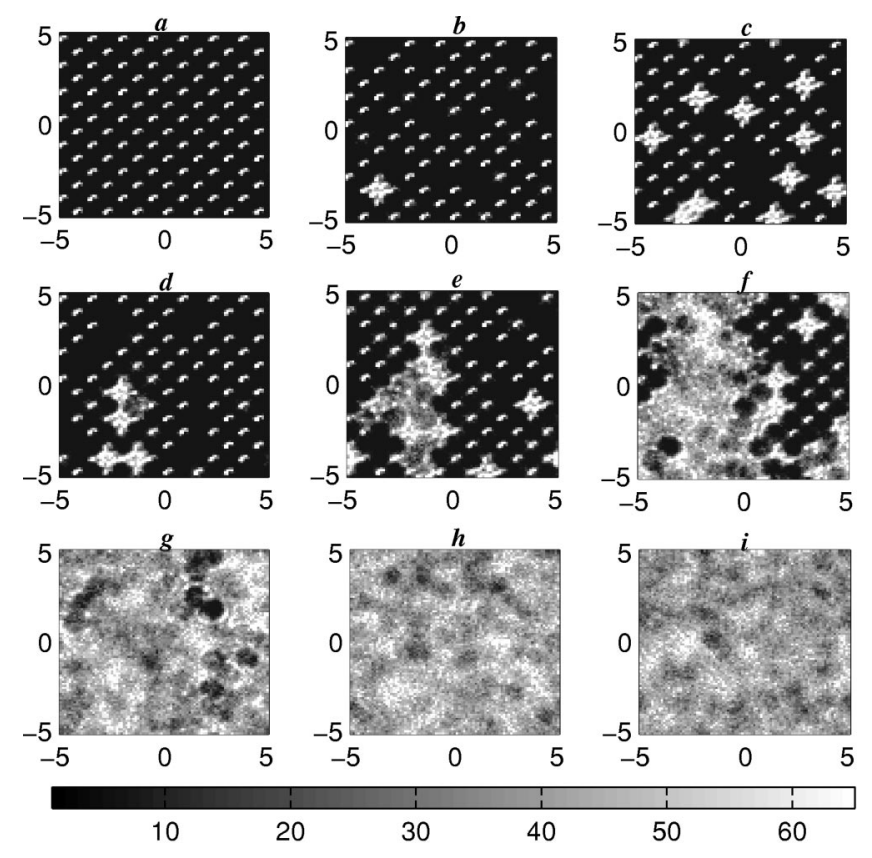

FIG. 7. Small particle density contour plots parallel to the interfacial plane for different layers of the [100] interface. The layers are as labeled in Fig. 3(a). 


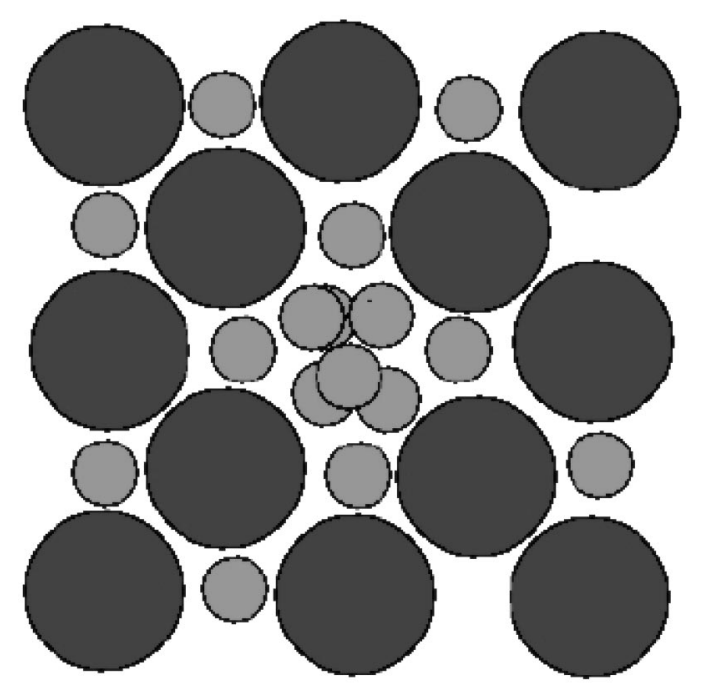

FIG. 8. Snapshot of a portion of layer $c$ as labeled in Fig. 3(a) showing small particles occupying a large particle vacancy.

for both particle types indicating that, although the $z$-dependent density profiles still has some oscillations in this region, the structure is that of an inhomogeneous fluid at a wall.

Of particular interest to materials scientists is the degree of interfacial segregation - the preferential adsorption (or desorption) of one component (usually the "solute") at the interface. This quantity is defined relative to the Gibbs dividing surface. The Gibbs dividing surface of a planar interface is defined ${ }^{1}$ as the plane along the $z$ axis giving a vanishing surface excess solvent particle number $\Gamma^{\text {solvent }}$ defined in the equation

$$
N^{\text {solvent }} / A=\rho_{c}^{\text {solvent }} z+\rho_{f}^{\text {solvent }}\left(L_{z}-z\right)+\Gamma^{\text {solvent }},
$$

where $N^{\text {solvent }}$ the total number of solvent particles spheres, $A$ is the area of the interface, $\rho_{c}^{\text {solvent }}$ and $\rho_{L}^{\text {solvent }}$ are the bulk densities, $z$ is the location of the interface assuming the length of the simulation box runs from 0 to $L_{z}$. Defining the small particles as the solvent, we find that the Gibbs dividing surfaces are at $z=-0.49 \sigma_{A}$ and $z=-0.93 \sigma_{A}$ relative to the position calculated from the large particle order parameter for the [100] and [111] orientations, respectively. The surface excess of the solute particles $\Gamma^{A}$ was found to be negligible, indicating the absence of interfacial segregation, a result that is consistent for other crystal/melt systems that have been investigated. ${ }^{7,9,13}$

\section{B. Transport: Diffusion coefficient profiles}

Inhomogeneities in the transport properties within the interfacial region can be examined by calculating $z$-dependent diffusion coefficient profiles, defined for a particle of type $i$ by

$$
D_{i}(z)=\lim _{t \rightarrow \infty} \frac{1}{6 N_{i}(z)} \frac{d}{d t} \sum_{j=1}^{N_{i}(z)}\left\langle\left[\boldsymbol{r}_{j}(t)-\boldsymbol{r}_{j}\left(t_{0}\right)\right]^{2}\right\rangle
$$

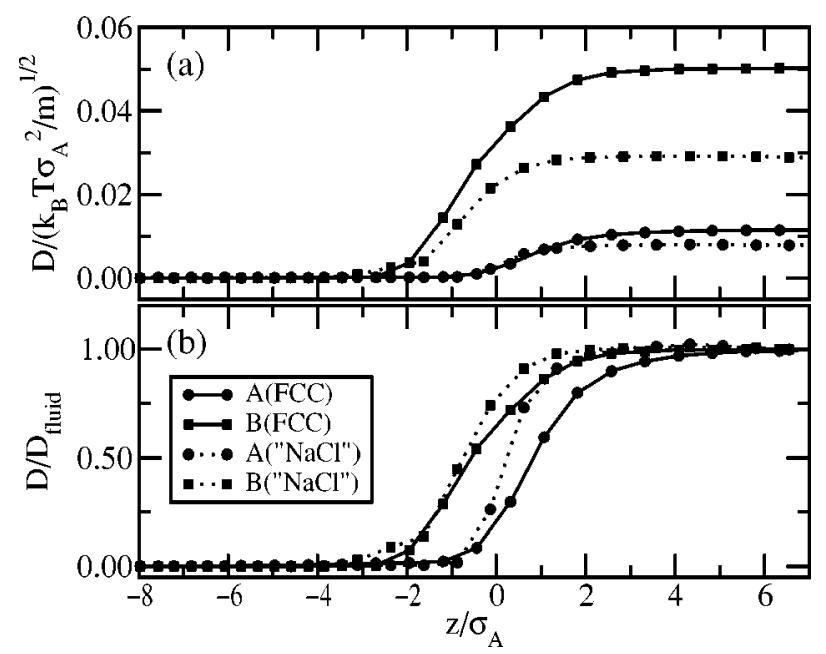

FIG. 9. (a) Diffusion coefficient profiles for the [100] orientation of the fcc/fluid (dashed) and the $A B /$ fluid (solid) interfaces for both particle types (circle for large and square for small); (b) Corresponding diffusion coefficient plots scaled to be zero in bulk crystal and unity in bulk fluid.

The term in the summation is the mean-squared displacement over a time interval $t-t_{0}$ of a total of $N_{i}$ type $i$ particles located between $z-\Delta z / 2$ and $z+\Delta z / 2$ at time $t_{0}$, where $\Delta z$ is the layer spacing in [100] and is twice the layer spacing in [111].

Figure 9(a) shows the diffusion coefficient profiles for the current study (dotted lines), including for comparison the results previously reported for the lower pressure fcc/binary fluid interface ${ }^{13}$ (solid lines). Only the [100] results are shown as the diffusion profiles for the [111] interfaces are not statistically different. The error bars are small and so are not shown for clarity of the plots. The bulk fluid value for the large particles in the lower pressure fcc/binary fluid system is $0.012\left(k T \sigma_{A}^{2} / m\right)^{1 / 2}$ and that for the small particles is $0.050\left(k T \sigma_{A}^{2} / m\right)^{1 / 2}$. Since the $A B /$ fluid system has a higher pressure and larger fluid packing fraction, the bulk fluid diffusion coefficient values are lower: $0.008\left(k T \sigma_{A}^{2} / m\right)^{1 / 2}$ for the large particles and $0.029\left(k T \sigma_{A}^{2} / m\right)^{1 / 2}$ for the small particles. The difference in magnitude between the small and large particle diffusion constants makes it difficult to compare the two diffusion profiles. For a clearer comparison in Fig. 9(b) we plot the data in Fig. 9(a) normalized relative to the bulk fluid values. Traversing the system from fluid to crystal, we find a region of width greater than $1 \sigma_{A}$ where the small particles have nonzero diffusion coefficient while the large particles have effectively zero diffusion. Both high- and low-pressure systems exhibit this shift in the change from crystal-like to fluidlike motion of the two particle types.

As was done for structural transition, we can also define the extent of dynamical transition by determining the 10-90 region from diffusion coefficient profiles. From the diffusion coefficient profile of the large particles this region starts from $z=-0.7 \sigma_{A}$ up to $z=1.3 \sigma_{A}$, resulting to a width equal to $2.0 \sigma_{A}$ and centered at $z=0.3 \sigma_{A}$. The small particles define an interfacial region that starts from $z=-2.2 \sigma_{A}$ and ends at 


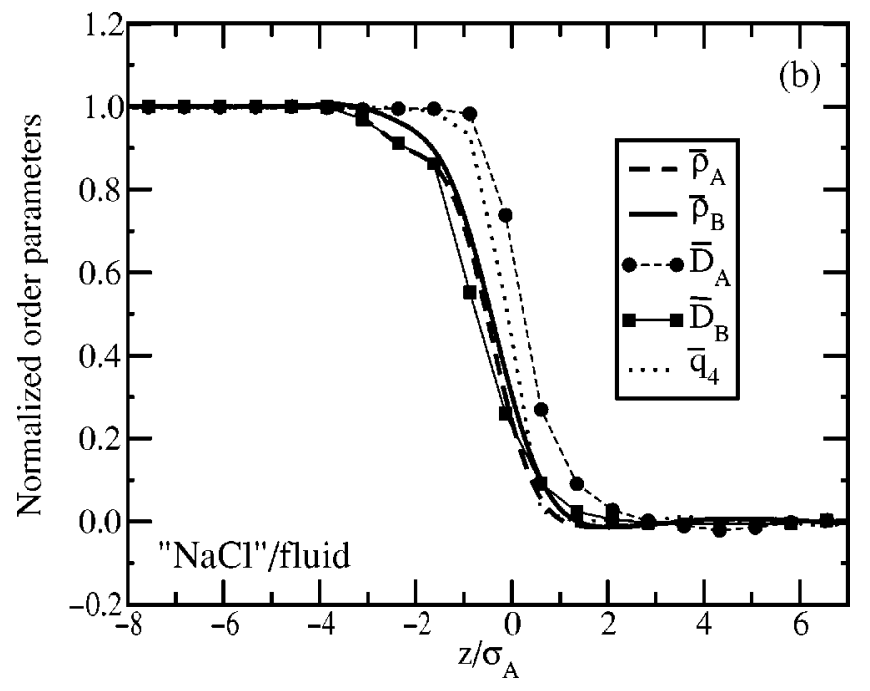

FIG. 10. Filtered density, diffusion, and orientational order parameter profiles for the [100] interface. All profiles are scaled such that they go from unity in the crystal to zero in the fluid phase.

$z=0.6 \sigma_{A}$. These boundaries give a width of $2.8 \sigma_{A}$, which is $40 \%$ greater than the width from diffusion of the large particles. Also, the midpoint is shifted by $1.1 \sigma_{A}$ to the fluid side relative to the midpoint of the region defined by the large particles.

As in our previous studies on hard-sphere interfaces ${ }^{7,13}$ the regions defined by each of the individual density and diffusion coefficient profiles do not all coincide, so we take a union of these 10-90 regions to define the full interfacial region. Taking into account all profiles, the $\mathrm{NaCl} /$ binary fluid hard-sphere interface studied here has an overall 10-90 width of $3.5 \sigma_{A}$, corresponding to layers $c$ to $g$ in Fig. 3. As perhaps expected for the higher pressure system, this interfacial region is narrower than the one found for lower pressure fcc/binary fluid interface, ${ }^{13}$ which was found to be $4.8 \sigma_{A}$. To summarize all of the profile data for quick inspection we show in Fig. 10 all order parameters profiles, normalized such that we have all values equal to unity in the bulk crystal and zero in the bulk fluid for the [100] interface. Except for differences in the overall interfacial width, this plot is qualitatively quite similar to the corresponding plot for our earlier low-pressure fcc/binary fluid simulations in that the transition of densities for both particle types and the diffusion of the small particles is observed over approximately the same region, while the transition for the large particle diffusion is shifted by about $1 \sigma_{A}\left(1.3 \sigma_{A}\right.$ in the earlier study). Another notable feature in this plot is that the transition for the orientational order parameter, which we use to locate the interfacial plane, occurs at about the center of these two transition regions.

\section{SUMMARY}

Using molecular-dynamics simulation, we have investigated the structure and dynamics of the [100] and [111] crystal-melt interfaces of the $A B(\mathrm{NaCl}) /$ binary fluid system for a two-component hard-sphere system in which the ratio of small to large particle diameter is $\alpha=0.414$. This system was at a pressure of $53 \mathrm{kT} / \sigma_{A}^{3}$ that is at the lower range of the $\mathrm{NaCl}$ crystal/fluid coexistence region. These simulations complement our earlier work ${ }^{13}$ on the pure fcc/binary fluid interface found in this same system at lower pressure $\left(20.1 k T / \sigma_{A}^{3}\right)$. We find that the higher pressure $A B /$ binary-fluid interface has a narrower interfacial region of $3.5 \sigma_{A}$ compared to the lower pressure fcc/binary fluid system at the same diameter ratio, which had an interfacial region of width $4.8 \sigma_{A}$. In contrast to both the lower pressure fcc/binary fluid system (and our previous single component ${ }^{7}$ and disordered fcc interfaces ${ }^{12}$ ), the peak spacing through the interface remained constant for both orientations studied. In addition, the crystal side of the higher pressure binary interface exhibited much higher vacancy defect concentrations than either the low pressure binary system or the singlecomponent interface. ${ }^{7}$ In the interfacial region, all vacancies in the large particle lattice were found, with little distortion in the surrounding lattice, to be filled with an average of six small particles. Similar to what was seen in the lower pressure fcc/binary fluid interface, the transition regions for both density profiles and the small particle diffusion constant are approximately coincident whereas the diffusion profile for the large particles is shifted relative to the others by about $1 \sigma_{A}$ toward the liquid side of the interface.

\section{ACKNOWLEDGMENTS}

We gratefully acknowledge R. L. Davidchack for helpful comments, as well as the Kansas Center for Advanced Scientific Computing for the use of their computer facilities. We also would like to thank the National Science Foundation for generous support under Grant No. CHE-9900211.
*Author to whom correspondence should be addressed.

${ }^{1}$ W.A. Tiller, The Science of Crystallization: Microscopic Interfacial Phenomena (Cambridge University Press, New York, 1991).

${ }^{2}$ B.B. Laird, in Encyclopedia of Computational Chemistry, edited by P.v.R Schleyer, N.L. Allinger, T. Clark, P. Kollman, and H.F. Schaefer (Wiley, New York, 1998).

${ }^{3}$ J.Q. Broughton and G.H. Gilmer, J. Chem. Phys. 84, 5749 (1986).

${ }^{4}$ J.Q. Broughton and G.H. Gilmer, J. Chem. Phys. 84, 5759 (1986).

${ }^{5}$ O.A. Karim and A.D.J. Haymet, J. Chem. Phys. 89, 6889 (1988).

${ }^{6}$ B.B. Laird and A.D.J. Haymet, J. Chem. Phys. 91, 3638 (1989).

${ }^{7}$ R.L. Davidchack and B.B. Laird, J. Chem. Phys. 108, 9452 (1998).
${ }^{8}$ J.A. Hayward and A.D.J. Haymet, J. Chem. Phys. 114, 3713 (2001).

${ }^{9}$ J.J. Hoyt, M. Asta, and A. Karma, Phys. Rev. Lett. 86, 5530 (2001).

${ }^{10}$ J.A. Hayward and A.D.J. Haymet, Phys. Chem. Chem. Phys. 4, 3712 (2002).

${ }^{11}$ R.L. Davidchack and B.B. Laird, Phys. Rev. E 54, R5905 (1996).

${ }^{12}$ R.L. Davidchack and B.B. Laird, Mol. Phys. 97, 833 (1999).

${ }^{13}$ R. Sibug-Aga and B.B. Laird, J. Chem. Phys. 116, 3410 (2002).

${ }^{14}$ M. Asta, J.J. Hoyt, and A. Karma, Phys. Rev. B 66, 100101 (2002).

${ }^{15}$ J.P. Hansen and I.R. McDonald, Theory of Simple Liquids, 2nd 
ed. (Academic Press, New York, 1986).

${ }^{16}$ D.A. Young, J. Chem. Phys. 98, 9819 (1993).

${ }^{17}$ B.B. Laird, J. Chem. Phys. 115, 2889 (2001).

${ }^{18}$ W.G.T. Kranendonk and D. Frenkel, Mol. Phys. 3, 679 (1991).

${ }^{19}$ E. Trizac, M.D. Eldridge, and P.A. Madden, Mol. Phys. 90, 675 (1997).

${ }^{20}$ X. Cottin and P.A. Monson, J. Chem. Phys. 102, 3354 (1995).

${ }^{21}$ M.D. Eldridge, P.A. Madden, and D. Frenkel, Mol. Phys. 79, 120 (1993).
${ }^{22}$ M.D. Eldridge, P.A. Madden, and D. Frenkel, Mol. Phys. 80, 987 (1993).

${ }^{23}$ M.D. Eldridge, P.A. Madden, P.N. Pusey, and P. Bartlett, Mol. Phys. 84, 395 (1995).

${ }^{24}$ D.C. Rappaport, The Art of Molecular Dynamics Simulation (Cambridge University Press, New York, 1995).

${ }^{25}$ W.H. Press, S.A. Teukolsky, W.T. Vetterling, and B.P. Flannery, Numerical Recipes in Fortran (Cambridge University Press, New York, 1992). 